\title{
Crowd Spatial Patterns at Bus Stops: Security Implications and Effects of Warning Messages
}

\author{
Réka Solymosi, Hervé Borrion and Taku Fujiyama
}

\section{Introduction}

As demonstrated throughout this book, the risk of certain types of crime can increase in congested spaces. Contact crimes, crimes which require the offender to make physical contact with the victim, are especially common in more crowded transport networks and can discourage many would-be passengers (Brand and Price, 2000). Pickpocketing makes up a substantial portion of this, accounting for around 50 per cent of all crime on London's transport network (Transport for London, 2012). Other chapters in this volume have emphasized the link between pickpocketing and bus stops, and this chapter will delve deeper into the mechanics of crowding at bus stops, and implications for pickpocketing and risk.

The aim of this chapter is to investigate crowding at bus stops by measuring micro-level spatial patterns of movements of individuals and consider implications for crime. Safety on the move is being approached on various scales in this book, and many meso- and macro-level studies reveal that risks are not equally or randomly distributed. This chapter takes a microscale approach, but replicates the findings from the above frameworks, to demonstrate that by observing the microlevel, detailed insight into where risks can occur could be generated.

To achieve new insight the chapter presents a novel approach of using a laboratory experiment as a method to study crime in a transport setting. This approach is used to measure fine details about interpersonal interactions at a crowded bus stop, and examine implications for interventions such as audio warning messages. The overall objective is to use insight into crowding gained by these experiments to make suggestions as to where future interventions should focus, and whether auditory warning messages provide a promising option, as well as to illustrate the potential benefits of this methodology for crime and transport research.

This article firstly reviews relevant literature on crowding and transport crime, pedestrian motion analysis and warning messages. It then presents

The original version of this chapter was revised:

This chapter was previously published with incorrect license type.

It has now been updated to CC BY 4.0. The correction to this

chapter is available at https://doi.org/10.1057/9781137457653_21. 
the research questions that arose from the reviewed literature. The experimental methodology is then presented in detail, followed by the results and discussion of the implications of the findings.

\section{Theoretical background and hypotheses}

\section{Transport crowding and theft}

Transport systems in large cities experience passenger volumes that test the higher end of their capacity. For example, in London, approximately 24.8 million total trips are made daily, using public transport (Transport for London, 2011). A peak time increase in ridership results in overcrowding, creating a criminogenic environment unique to transport environments (Smith and Clarke, 2000). In a crowded environment, contact crime, that is, crime that thrives where people come into close contact with one another, can present a significant problem. Crowding is a precondition of contact crime (Smith and Clarke, 2000), and typical offences include theft (Kabundi and Normandeau, 1987) and sexual assaults (Beller et al., 1980). Indeed many of the chapters in this book focus on the relationship between crowding, including increase in theft risk with congestion, and the impact of passenger flow on pickpocketing at bus stops.

At the meso- and the macro-levels, observational studies have demonstrated how crowding specifically at stations and stops has been associated with high rates of crime and fear of crime (Shellow et al., 1974; Kenney 1986). Pickpocketing in particular occurs at overcrowded stops at which offenders can take advantage of the high densities of people who are close together (Loukaitou-Sideris and Liggett, 2000, Loukaitou-Sideris, 1999, Liggett et al., 2001; Loukaitou-Sideris, 2012; Loukaitou-Sideris et al., 2001). Additional studies which examine information from police files, revisit sites and interview offenders and victims suggest that the specific act of pickpocketing frequently takes place during boarding, when people are getting on the bus, and are crowding around the bus door, rather than while waiting or after boarding the bus (Poyner, 1986). While singling out a specific element of the entire process of crowding at bus stops may help target interventions, there is not enough insight into the mechanisms of crowding to justify this. Therefore this chapter will study the spatial patterns of crowding in more detail, to attain further insight into what happens while people are boarding a bus.

\section{Bottlenecks}

Research on pedestrian motion analysis has found that often, in crowded environments problems emerge due to bottlenecks (Helbing et al., 2005). Bottlenecks are areas in which there is a significant capacity drop in pedestrian movement, such as a narrow doorway in a corridor, where jamming occurs when the incoming flow exceeds the capacity of the bottleneck (Seyfried et al., 2009). Hoogendoorn and Daamen (2005) observed that 
during such a jam, pedestrians form layers, and while trying to navigate through a door one metre wide, the width of these layers will become less than the effective width of a pedestrian, causing them to overlap. Effectively, when passing through a bottleneck, people come in close proximity, effectively moving into each other's personal space, which enables close contact such as getting within arm's reach of one another.

Assuming that the bus door causes capacity to drop and forces people to come within close distances of one another while boarding, this would create many opportunities for one person to reach into the pocket of another. If the bottleneck at the bus door has such an effect on the crowd's movement, we should see people coming closer to one another, and doing so more frequently. Identifying the bus door as a bottleneck that produces such an effect can help shift focus to that specific element of crowding at bus stops and inform better planning interventions to reduce this. Laboratory experiments are a common methodology for examining pedestrian behaviour in bottlenecks. They have the advantage of creating a controlled environment in which variables related to spatial patterns can be measured. Studying micro-level interactions in a laboratory setting results in many findings and real-world applications (for an example see Helbing et al., 2005), for crime prevention practitioners, as well as academics working on meso- and macroscale models, or academics from other disciplines interested in using pedestrian motion analysis to study social phenomena.

\section{The self-organizational behaviour of crowds}

Routine activity theory states that crime occurs given the intersection of a likely offender, a suitable target and the absence of a capable guardian (Felson and Cohen, 1979). To prevent and control crime, we can attempt to manipulate one of more of these three variables. This chapter will focus on how crowding can produce suitable targets, and will assume the presence of a motivated other. Crowding is affected by density (the number of people using the bus) and by the width of the bottleneck (bus door). However, reducing the number of people using the transport system or widening all bus doors is an unrealistic solution. Alternatively, other factors also influence crowding in a bottleneck, such as the behaviour of passengers passing through (Hoogendoorn and Daamen 2005). People's behaviour is influenced by their environment (Evans 2009). For example, similar to crowding at bus stops, crowding at nightclubs has been associated with increased crime rates (Macintyre and Ross, 1996). Yet Macintyre and Ross (1996) established a difference between 'good' crowding and 'bad' crowding, independent of density, suggesting better design standards for nightclubs, shifting focus from crowd control and capacity management approaches to the layout and design of clubs. Similarly, an intervention that would encourage pedestrians to adopt self-protective behaviour while boarding a bus could help reduce crime at bus stops. 
To observe self-organizational behaviour, this project focuses on the microlevel interactions between persons in a crowd. Pedestrians' movements are affected by interactions with other pedestrians (Helbing et al., 2001). The term 'micro-level interaction' refers to local interactions, from which collective behaviour emerges (Moussaiid et al., 2009). Goffman (1971) found that pedestrians react only to those other pedestrians who are in a small circle around them, neglecting others who are one or two persons away. Therefore, to study the micro-level interactions in a crowded setting, it is important to focus on those persons immediately surrounding the individual, and the relationship between them, as those individuals further away (more than two persons away) will not affect their actions. Thus the study will focus on the micro-level interactions within the context of crowding at bus stops.

\section{Warning messages}

Warning messages are an example of an intervention currently in use, aimed at encouraging passengers to adopt self-protective behaviours (Metropolitan Police 2011; British Transport Police 2011). The purpose of a warning is to alert people to potential hazards (Stewart and Martin 1994) and to encourage modification of behaviour to protect against them (Wogalter and Laughery 1985). However, often they are informative rather than persuasive, and can be ignored, reducing their impact as a preventive measure (Jacoby et al., 1998). Even if attended to, warning messages may have unintended consequences, like serving as a tool for pickpockets; posters can be used to pinpoint valuables when people walking by them tap their pockets to check if they still have their belongings (see Ekblom, 1995). During a passenger journey on public transport, the primary goal is to reach one's destination. To ensure a message is observed can be especially challenging in this context, and the extent to which passengers' behaviour can be altered is furthermore restricted by features of the environment. Applying methods from crowd dynamics, mentioned earlier, may serve to answer questions about the feasibility of using warning messages to encourage change in behaviour in restricting environments.

\section{Research questions}

The research questions were devised to be feasible within the simplified context of a laboratory setting, yet still produce meaningful insight into crowding behaviour relevant to a bus stop. Based on the identification of the boarding component of the bus journey, as the phase in which pickpocketing takes place (Poyner, 1896), and on literature on pedestrian motion analysis, discussed above, it can be speculated that the creation of a bottleneck at the entrance of the bus amplifies the crowding situation. To determine whether this is the case, research question one asks, is there 
a difference in the distance passengers keep from those immediately around them between when they are waiting, boarding and being on a bus?

H1: There is a difference in the distance people keep from one another at the different phases of the transit journey (waiting at the stop, boarding the vehicle and being on a bus)

The above literature summary also suggests that altering passenger behaviour within a crowded environment may reduce 'bad crowding'. Question two will seek to determine whether people are capable of altering their behaviour in terms of distance from those immediately around them, when exposed to auditory warning messages about the presence of a pickpocket, despite having to carry out their primary goal of completing a bus journey.

$\mathrm{H} 2$ : There is a difference in the distances people keep from one another between control, warning and pickpocket scenarios.

Answering these questions will identify what specific phase of crowding is 'bad crowding' (when people come close enough to be able to reach one another), and whether spatial behaviour on the micro-scale can be changed

\section{Method}

Most literature on crowding, bus stops and pickpocketing relies on field observations, providing valuable and detailed insight into contact crime in transport environments from this angle. To gain new insight, a laboratory experiment was chosen for this study, to address field studies' difficulty in measuring concepts and altering or controlling variables (Eck and Liu, 2008). The simplification of context allows for measurement of microlevel interactions that make up crowding by observing interpersonal distances during the process of waiting for, boarding and finding a place on a bus. This choice of methodology serves to introduce something new into research on contact crimes in transport environments. Further, the direct relation of the research questions to the simplified context makes the laboratory experiment a useful tool for observing rules and patterns of pedestrian behaviour and group self-organization (Helbing et al., 2005; Daamen et al., 2008). Practical reasons such as the ability to precisely measure positional data and run replicable scenarios that are not available or difficult to observe in normal conditions (Hoogendoorn and Daamen, 2005) are a further benefit of choosing a laboratory experiment as the method for this study.

The experiment was carried out at University College London's (UCLs) Pedestrian Accessibility Movement Environment Laboratory (PAMELA). PAMELA has been used to study how pedestrians navigate urban spaces (Cepolina and Tyler, 2005; Fernándezet et al., 2010; Fujiyama and Childs, 2005). A mock-up bus shelter and bus were constructed in PAMELA to replicate the study environment. Measurements for the shelter were initially 
determined by consulting guidance manuals (Transport for London, 2006), but were later adjusted based on measurements of actual bus shelters in North London, to ensure real-world parameters. A full scale 'mock-up' of the front section of a New Bus for London (NBfL) bus was built to correspond with the exact parameters of NBfL. Only the front section was constructed to ensure that the crowding level (see Participants section) was maintained throughout the experiment. It is assumed that the bus is so crowded that participants can only fit into the front section of the bus.

Ambient traffic noise and sounds representing a bus pulling up to a stop and opening its doors were played over phase array speakers in PAMELA to provide an appropriate background (Childs et al., 2005). A range of environmental factors were included, such as vertical and horizontal gaps, door width and internal arrangement of space, to replicate those of a real bus, in order to recreate the type of bottleneck effect that would occur during boarding in the real world. Appendix 9A shows the final parameters and set-up of the experiment.

To measure distances between individuals, participants' movements were tracked by motion-tracking devices and studied in relation to one another. The motion trackers consist of small wireless markers (Appendix 9B) that record Cartesian coordinates of their location in reference to a coordinate grid set out by sensors (Appendix 9C) with which they communicate using radio frequency, at a frequency of $47 \mathrm{~Hz}$ (recording data 47 times per second). The markers were placed on hard hats, which participants were instructed to wear (Appendix 9D). The sensors were set-up around the experiment environment (Appendix 9E).

The distance between markers was obtained using the following equation:

$$
d=\sqrt{\left(X_{B}-X_{A}\right)^{2}+\left(Y_{B}-Y_{A}\right)^{2}+\left(Z_{B}-Z_{A}\right)^{2}}
$$

The above procedure was used to find the distances between all marker pairs for the full duration of each run of the experiment. All three coordinates were considered in order to get the absolute distance between markers. If any shift in the coordinate grid occurred due to participants' accidentally bumping into the infrastructure, the distances measured between markers would still remain consistent, allowing for comparability between all the scenarios and experiment runs.

A total of 16 participants were recruited with a mean age of 25.4 years, with the youngest at 19 and the oldest 40 years old. There were 10 male and 6 female participants. Most participants were strangers to each other, although there was one group of three who were friends who moved together as a group. To create a more diverse range of boarding behaviour, participants were assigned roles which they were instructed to perform 
during the course of the entire experiment. Roles were assigned to participants randomly, and contained information such as the urgency with which they needed a seat (passenger who wants a seat on the bus, elderly passenger, passenger in a hurry and normal passenger) to replicate the competition for space that takes place in a real bus environment. Participants were also asked to keep their roles secret from each other. Enough participants were recruited to recreate crowding in this portion of the bus and at the mock-up bus shelter. This number was achieved based on findings from behavioural experiments on personal space preferences. Minimum desirable occupancies range from five to ten square feet per person, and the experimental set-up provided significantly less than that, to achieve crowding (Fruin, 1992).

\section{Experiment procedure}

For the experiment, participants were asked to wait at the mock-up bus shelter for about one minute, after which they were prompted to board the bus and situate themselves on it. This was repeated nine times. After the every third repeat, the warning scenario condition was changed. The three different scenarios will be referred to as control, warning and pickpocket. The control scenario consisted of participants waiting at the bus stop and boarding the bus when indicated. The warning scenario was the same as the control, except alongside the noise of traffic and the bus, a standard station announcement, recorded from a UK station, was played over the sound system (automated voice) while the participants waited at the bus stop. The recorded message said, 'May I have your attention please: would customers please note that pickpockets operate on this station. Please do not leave any item of luggage unattended at any time. Please make sure your personal items are secure'.

In the pickpocket scenario, the participants were informed by the experimenter, that one of them has been given the role of pickpocket. It was verbally explained by the experimenter (human voice) that the pickpocket's task was to place a playing card on the person of a fellow participant. In reality, none of the participants were actually given this role. The aim was to encourage vigilance amongst participants, providing the most serious warning condition. Participants were told to aim to avoid becoming the victim of the 'pickpocket'. This scenario is much like the popular game 'assassin', in which players have to come close to one another undetected to place an object on the other person (for example, a sticker or a playing card). As it was not possible to hire a professional pickpocket, or for the participants to believe one of them was an actual pickpocket, this was a believable alternative which the participants understood as a credible threat. For every run of the experiment, participants spent roughly the 
same amount of time waiting at the bus stop before they would board the bus. Data recording was stopped after all participants had boarded and found a place on the bus.

In order to measure only micro-level interactions between participants, and to focus on those who came 'close enough' for a pickpocket to physically be able to operate, people's peripersonal space was considered. This is the space immediately surrounding a person, within which objects can be reached without the person's moving (Holmes and Spence, 2004). To operationalize this measure, a study of arm-reach carried out to inform fighter-jet design was consulted (King, 1948), which found this threshold distance to be 26.7 in $(67.8 \mathrm{~cm})$. The following sections will detail the results of the experiment, analyse them in terms of the research questions and present a discussion of the findings.

\section{Results}

Initially, the $\mathrm{x}$ and $\mathrm{y}$ coordinates of participants were plotted to show their trajectories for the first run of the experiment (Figure 9.1).

As individual trajectories describe where participants went in space, but not much about their relation to one another, this data was further analysed

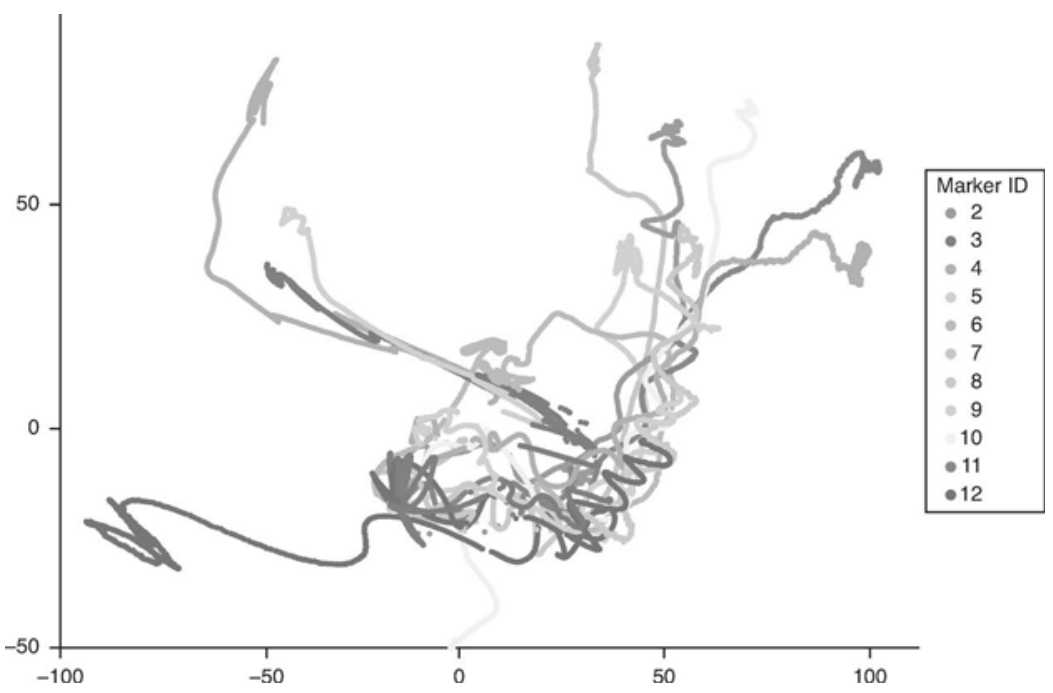

Figure 9.1 Trajectories of participants in one run of the experiment showing their movement while waiting for boarding and dispersing from the mock-up bus 
to calculate the distances between people. Firstly, the smallest distance between people during each phase (waiting, boarding, on bus) of each run (control, warning and pickpocket) was considered. Figure 9.2 shows a frequency distribution of minimum distances. Each smallest distance per marker pair was plotted on a histogram, in which the $\mathrm{x}$ axis shows distance in inches, grouped into categories (those that fall between 0 to 10.0 in, 10.1 to $20.0 \mathrm{in}$, etc.), and the height of each bar represents the number of values that fall within each group. This data is normalized for the number of valid measurements collected. Therefore, the data presented is the proportion of the valid samples that fell within each distance category.

During boarding, the closest people came to one another was most frequently between 0 and 20 inches. This is very different from the waiting and on the bus phases, in which a more even distribution is present. More people come very close to one another during the stage of boarding than the other phases of 'waiting' and 'on the bus'. Indeed, looking at all nearest distances between pairs, on average people maintained a minimum distance over twice as large while 'waiting', or 'on the bus', than what they managed to keep during 'boarding'.

As this data does not follow a normal distribution, a Wilcoxon signed rank test is used (Baguley, 2012) to determine that this difference in minimum distances kept is significant between the waiting, boarding and

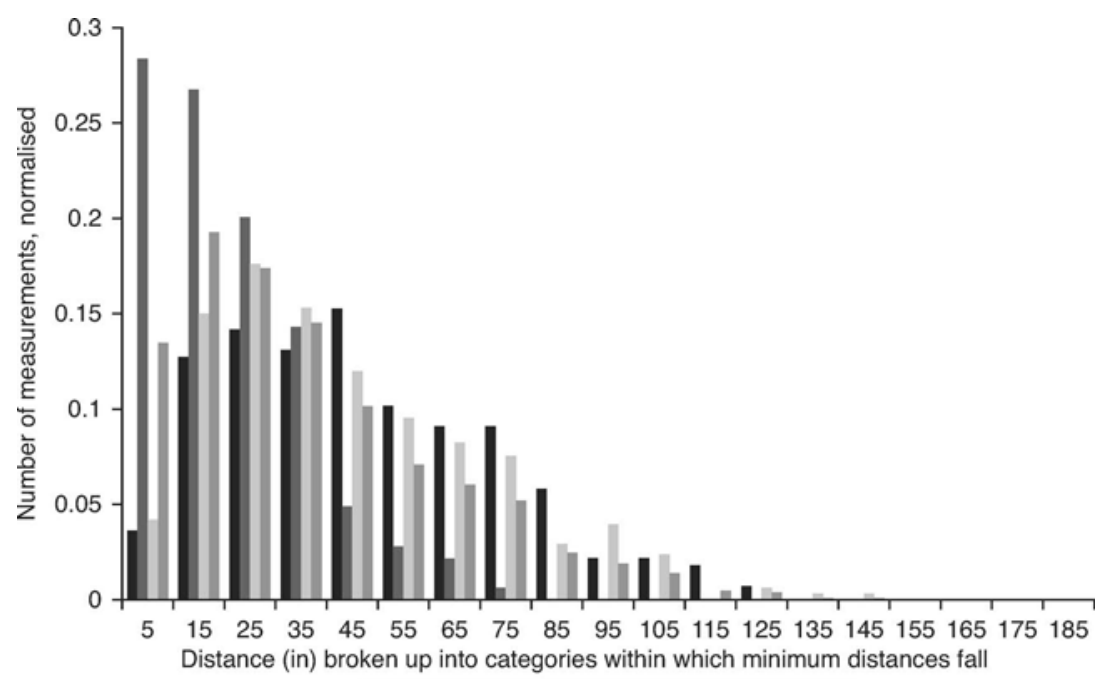

Waiting $\mathbf{m}$ Boarding $\mathbf{m}$ On bus $\mathbf{m}$ Overall

Figure 9.2 Distribution of the smallest distances between people broken down by phase 
on the bus phases $(\mathrm{p}<0.05)$. The bus door does indeed create a bottleneck in which people are forced significantly closer to one another. Looking back at people's trajectories within the experiment space, Figure 9.3 shows participants coming together and then moving apart during boarding.

To determine opportunities in which participants would be able to reach each other's pockets, we refer back to the measure of peripersonal space. Figure 9.4 shows how many times two people came within arm's reach of one another (normalized for number of valid measurements). Of all the phases, it was while boarding that two people were most likely to come close enough to one another that one could reach the other's pockets. The bottleneck effect created when passengers board the bus clearly forces people closer together, more often than other stages of crowding at bus stops. This indicates that 'bad crowding', when people come close enough to be able to reach another, is most likely to occur when people are boarding the bus.

However, time spent within arm's reach is another important factor to consider. If two people move within each other's peripersonal space (threshold distance), but merely bump into one another and move away from this immediately, a person may not have been exposed to any threats such as pickpocketing because this act may require a longer time frame. A further element of people's behaviour in terms of interpersonal distance is the length of time they spend within another's peripersonal space. As the length of time each experiment took was varied, due to the versatile nature of a large group of people boarding a bus, measurements of time were normalized for comparability. Therefore, the following time data are presented not in seconds, but in seconds per minute. So, for example, if the phase lasted precisely one minute, the seconds per minute would give the exact number of seconds spent within threshold.

To see where measurements fall, a graph similar to those used for distance measurements was plotted. Figure 9.5 shows how long participants who came within arm's reach of one another stayed there before moving away. Bar height represents how many measurements fall into any of these categories. Bars represent the three phases of waiting, boarding and on the bus. When two people never came within threshold distance to one another, this would obviously result in ' 0 ' seconds, and these were excluded from this analysis.

Boarding and on the bus measurements fall most frequently into categories in which people move away from each other between 0 and 30 seconds per minute after initially coming within arm's reach. However, the majority of those who came within threshold distance of one another during waiting phase fall into the 55-60 second category. This means that those who stood within arm's reach of one another while waiting for the bus stayed there for up to the entire duration of the phase. The difference between how long 


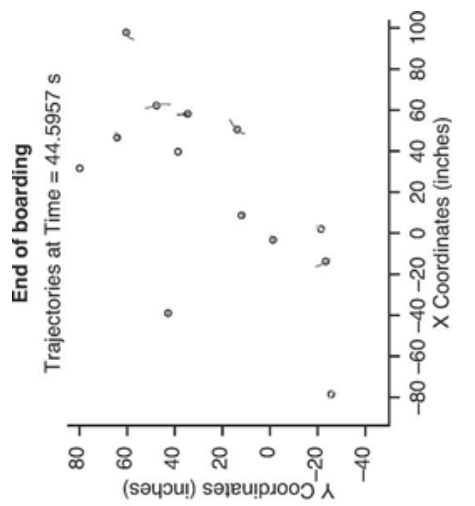

苞

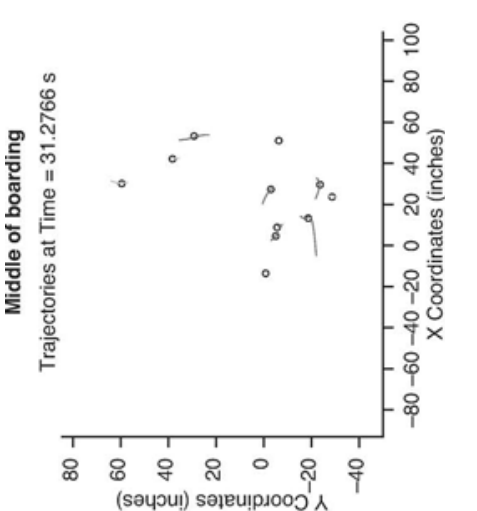

ב

$\stackrel{b}{\Xi}$

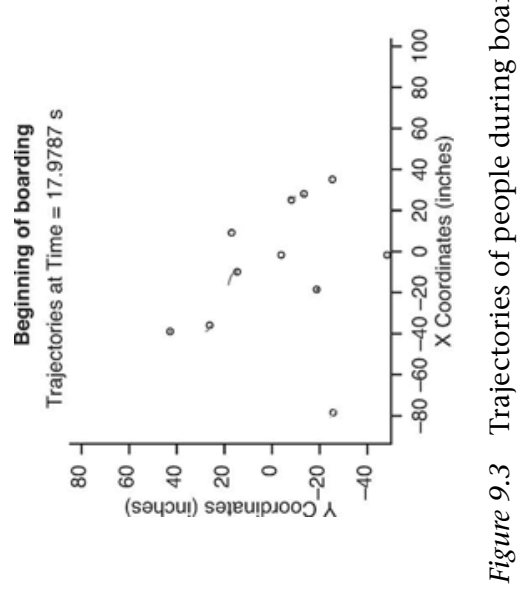




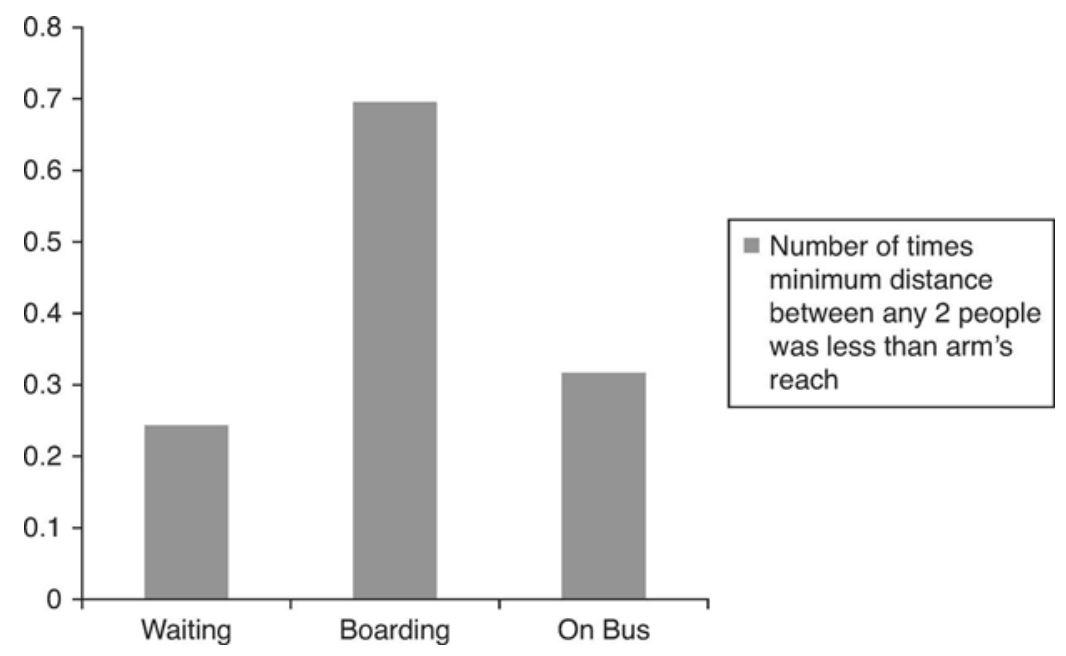

Figure 9.4 Number of times minimum distances between people were less than threshold per phase

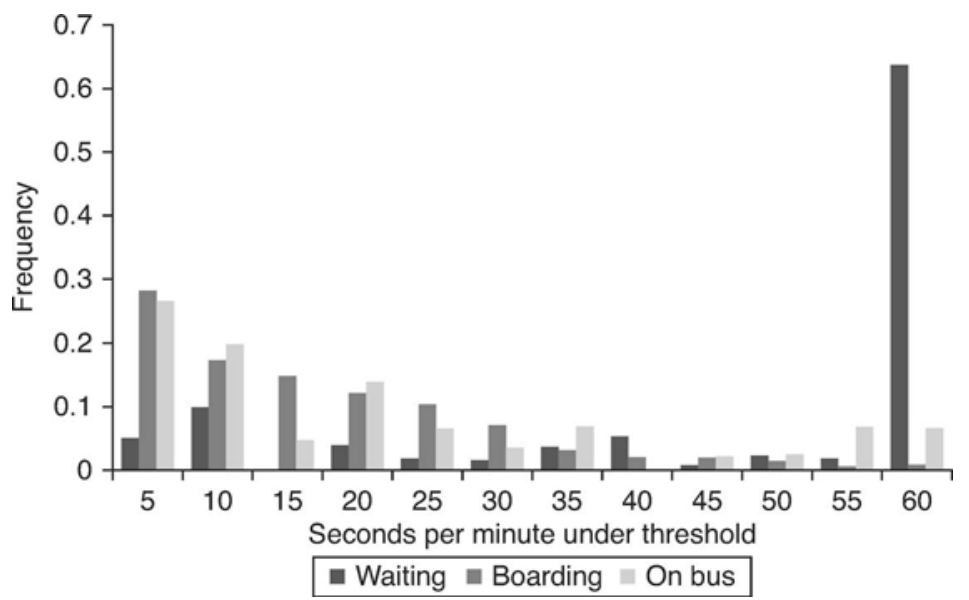

Figure 9.5 How much time people spent within threshold distance of another, by phase

people spent within threshold during warning and the other two phases is statistically significant (Table 9.1)

The amount of time spent close to others also shows a noticeable difference during the waiting phase. Findings show that if we consider length of time spent within arm's-reach of another as an important factor as well as the 
Table 9.1 Wilcoxon signed rank test for difference between duration of time people spend within arm's reach distance per phase of experiment

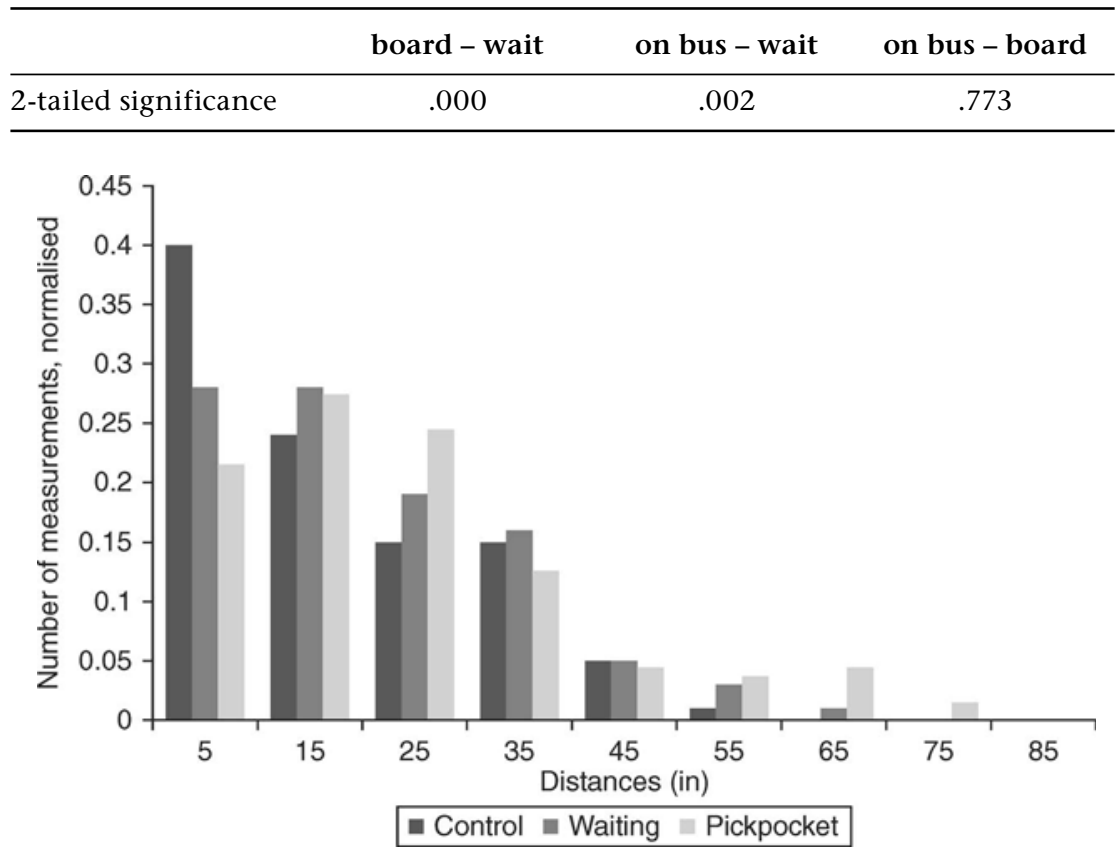

Figure 9.6 Minimum distances (in) by warning scenario, during boarding

number of times a person can be reached, waiting at the bus shelter might provide opportunities for contact crimes as well as the boarding phase.

Given the micro-level spatial interactions in which people come within arm's reach of one another in the context of waiting for and boarding a bus, if motivated by audio warning messages, can they alter the distance they keep from fellow passengers? To answer this question, minimum distances during boarding were considered for each warning condition. In a similar graph to Figure 9.2, distribution of smallest distances was plotted for all three scenarios (Figure 9.6).

Figure 9.6 shows that minimum distances fall mostly into the smallest category during the control scenario, and shift slightly towards the larger distance categories in both the waiting and the pickpocket scenario. It is also in the pickpocket scenario (most serious warning condition) that the largest minimum distance is observed.

The Wilcoxon signed rank test reveals that there is a significant difference between control and pickpocket scenarios in the closest distances that people got to one another at a $\mathrm{p}<0.05$ level Table 9.2. 
Plotted in Figures 9.7, 9.8 and 9.9 are the cumulative distribution functions, $\mathrm{F}_{\mathrm{T}}(\mathrm{t})$, of the time (t) spent within threshold distance, to show the proportion of measurements that fall below the threshold. This illustrates that during waiting, in the pickpocketing condition there is a higher probability of people spending less time within threshold distance of one another

Table 9.2 Wilcoxon signed rank test statistic for minimum distances while boarding

\begin{tabular}{lccc}
\hline & $\begin{array}{c}\text { warning - } \\
\text { control }\end{array}$ & $\begin{array}{c}\text { pickpocket - } \\
\text { control }\end{array}$ & $\begin{array}{c}\text { pickpocket - } \\
\text { warning }\end{array}$ \\
\hline significance & .158 & .000 & .001 \\
\hline
\end{tabular}

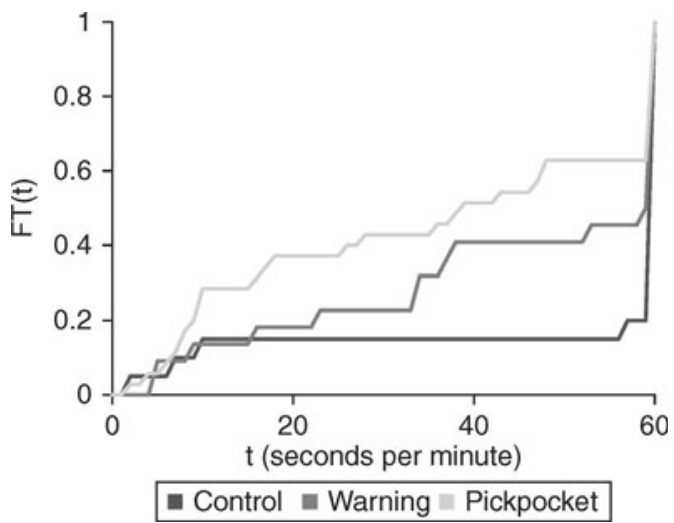

Figure 9.7 Cumulative distribution of the time under the threshold for waiting

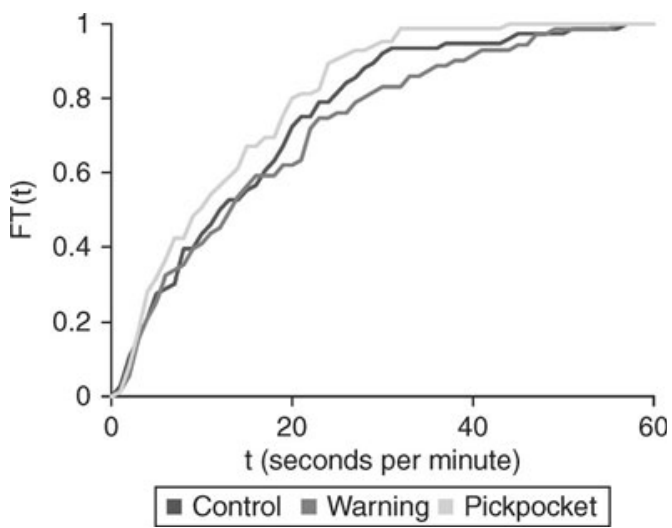

Figure 9.8 Cumulative distribution of the time under the threshold for boarding 


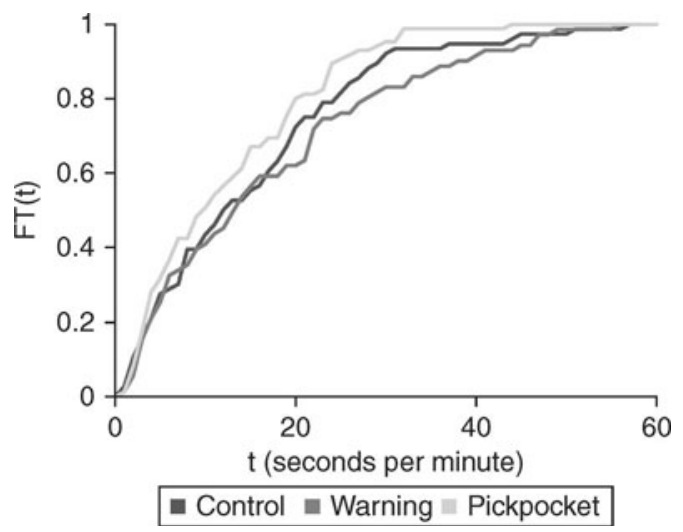

Figure 9.9 Cumulative distribution of the time under the threshold on bus

than in warning or control conditions. However, these differences are not statistically significant.

\section{Discussion}

Results from this experiment indicate that a bottleneck is created when people board the bus, in which they come much closer to one another than before (waiting) or after (on bus) (Figure 9.2) and come within arm's reach with many more passengers than they do while waiting for the bus or when aboard the bus (Figure 9.4). Considering a passenger's coming close enough to another person for him or her to be able to reach that passenger's pocket as creating an opportunity, this finding supports previous theories that pickpocketing opportunities are provided when individuals are boarding the bus (Poyner, 1986). This has practical implications for prevention to shift focus onto boarding.

For example, closed-circuit television (CCTV) cameras on buses tend to focus on the inside of the bus, so directing some to cover the area in which people board the bus may be a possible intervention to consider. Other situational crime prevention measures aimed at reducing the bottleneck effect could be developed through future research that experiments with different queue marshalling barriers (Poyner, 1986) or design techniques which influence jams at bottlenecks (Helbing et al., 2005). These could also address other proximity-related crime such as groping. Furthermore, the finding that the jam caused by boarding the bus exhibits similar crowd movement patterns to other unilateral bottlenecks, implies that findings 
from pedestrian motion analysis could inspire future research on crime related to crowding at bus stops.

However, it is important to note that even though a bottleneck phenomenon during boarding may cause more people to come closer to each other than during other phases, this may not be the only variable to consider. Looking at the time that people spend within arm's reach of at least one other passenger reveals that while people come closer together more frequently during boarding, they do not spend a lot of time within this threshold. This may be due to the nature of the activity of boarding a bus; people are constantly moving trying to get on the bus. During the waiting period, people do not move as much. If two passengers are near one another, they will remain so until there is a reason for them to move. Further research should look into a time threshold for pickpocketing, and determine whether increased time spent close to one another during the waiting phase increase exposure to potential pickpockets, and also whether the time spent very close to one another while boarding is long enough for a contact crime to occur.

Findings also indicate that people are capable of modifying and willing to modify their behaviour within the crowded environment in light of audible warning messages. People showed a tendency to keep larger distances from one another within the bottleneck environment when hearing the most relevant and credible warning message of the experiment (Figure 9.5). This may have positive implications for the use of audio warning messages at stations to warn passengers. Contents of warning messages was not a topic of this research, and future research might focus on the feasibility of creating a credible and attention grabbing warning, applicable to realworld environments. Additionally, the finding that people do not move around while waiting for the bus can have implications for anomaly detection surveillance programs. If this pattern is found to be consistent with real-life observations, this could inform CCTV pattern analysis, used in a variety of security applications such as counterterrorism strategies (Davies and Velastin, 2005).

Additionally, results that pertain to individuals' micro-level behaviour and interactions with one another and their environment can inform models used in pedestrian simulation related to security and crowd control. Modelling provides a useful methodological tool for attaining rigorous results from large datasets (Antonini et al., 2004; Scholl 2001; Teknomo 2002; Wijermans et al., 2007; Yavuz et al., 2007). Agent-based models (ABMs) look at the global consequences of local interactions by using agents, which are assigned a small set of rules which govern their behaviour (Scholl 2001) and are used in a variety of academic disciplines. ABMs have many components, one of the most important of which is the rules given to the agents by which 
to act (Macal and North, 2010). This experiment can help calibrate and validate such models by adding to the empirical evidence based on real people's actual behaviour, which may inform rules assigned to agents (Helbing and Balietti, 2011).

This chapter has demonstrated the use of data collected in a laboratory experiment to gain further insight into measurable variables related to a criminological problem. While the benefits, including the ability to control and manipulate variables, and produce repeated measurements have been illustrated throughout this chapter, there are some important limitations to mention. The homogeneity and size of the participant group may not accurately represent all passenger characteristics. While this was addressed by assigning roles to participants to diversify their behaviour, a repeat of the study with a more heterogeneous group may provide more generalizable findings. Additionally, the effect of order bias (Landon, 1971) may have had an influence on differences in measurement as the experiment progressed. Additionally, while participants filled the experiment space to a crowded level as defined by Fruin (1992), levels of crowding can be intensified by external variables such as punctuality of the bus, or if a bus is running late. Future research might look into more intense levels of crowding as well as other variables present in a real-life setting, such as distractions caused by headphones, and how these might affect the observed behaviours. In this experiment the recording equipment was constrained by the availability of markers (there were only 11 active markers to be used at one time). However, the data analysis took into account this limitation and consisted of comparing data on distances between recorded participants within immediate range of one another. If all participants were recorded, additional analysis could have been achieved, for example, density calculations. However, in the absence of this it is unknown whether non-measured people would have an effect. Finally, the laboratory offered a risk-free environment for participants which may have affected their perception of the credibility of warning messages. To address this, the pickpocket scenario was developed to be believable and relevant within this context.

Evidently there are limitations associated with such a laboratory experiment; however, 'basic research in a controlled laboratory environment is a necessary first step to identifying effects that subsequently can be tested in a field context' (Schultz and Tabanico, 2009: 1205). This chapter attempts to demonstrate a way to draw in knowledge and techniques from other research areas to the topic of crime and transport, and broaden the range of this topic by providing a stepping stone to achieving such results and offering an additional lens through which further insight can be gained. 


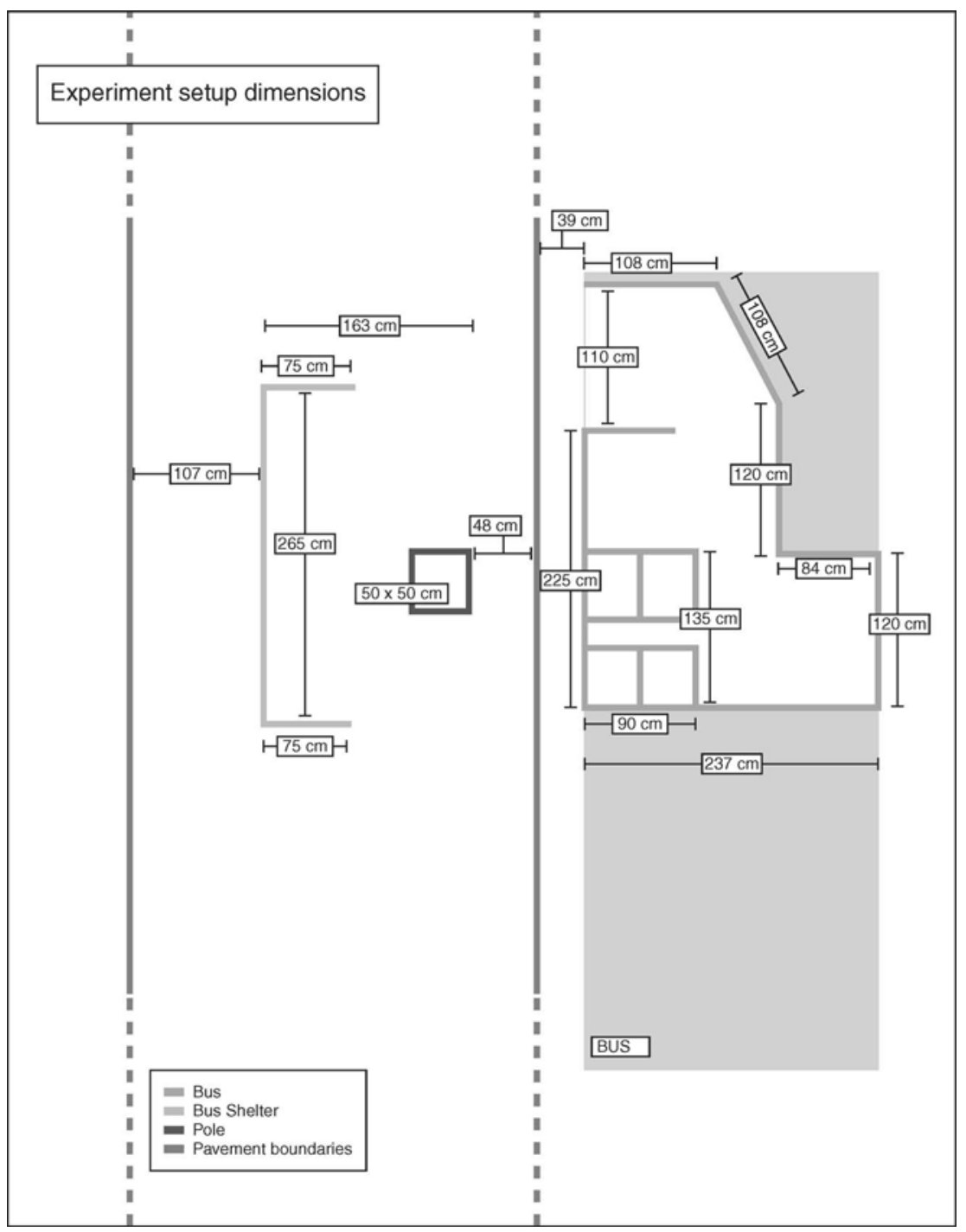

Appendix 9A Dimensions of the recreated bus waiting area and bus environment 


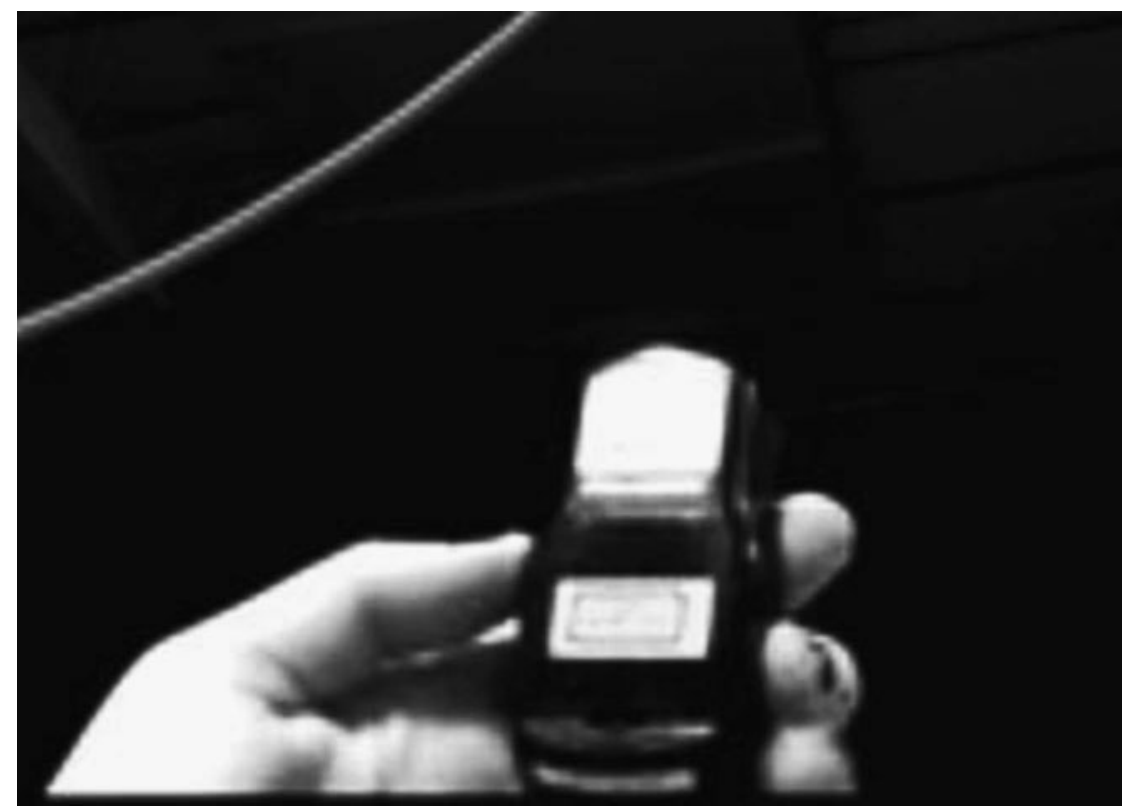

Appendix 9B Wireless marker

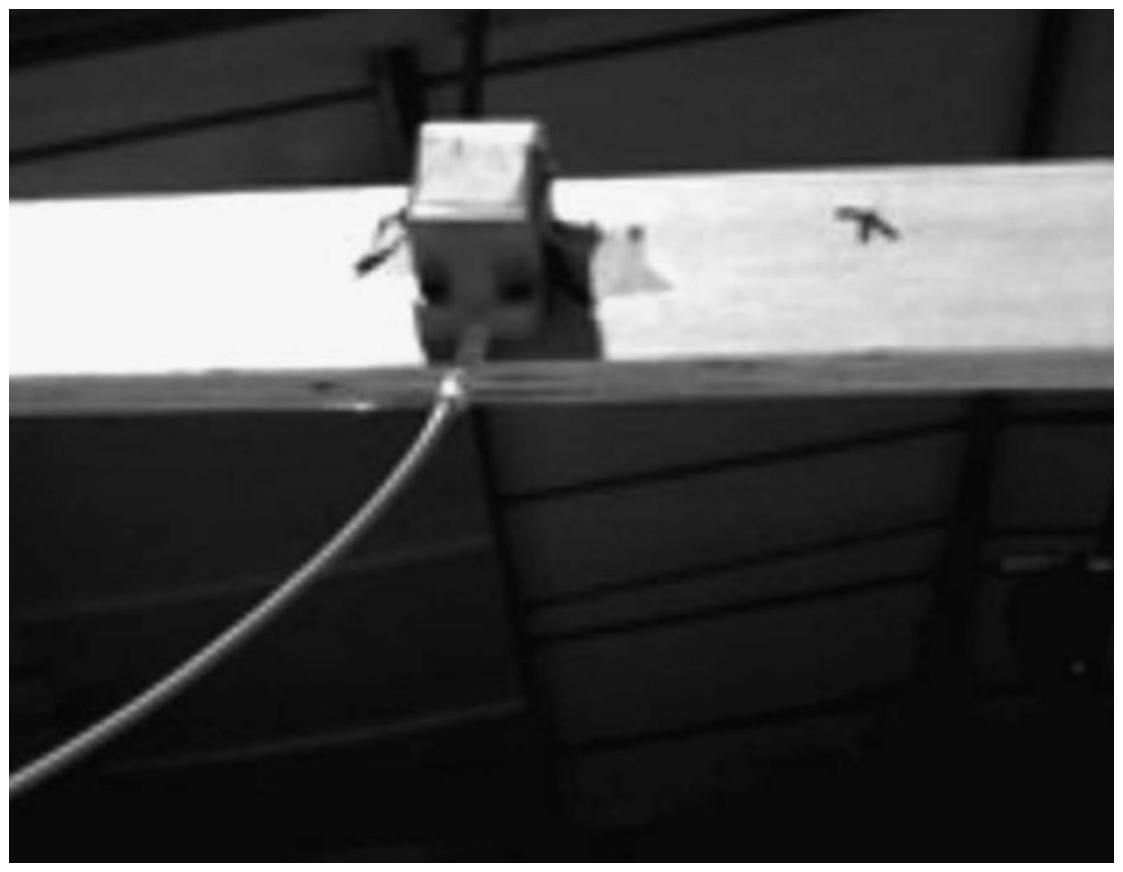

Appendix 9C Sensor-defining grid 


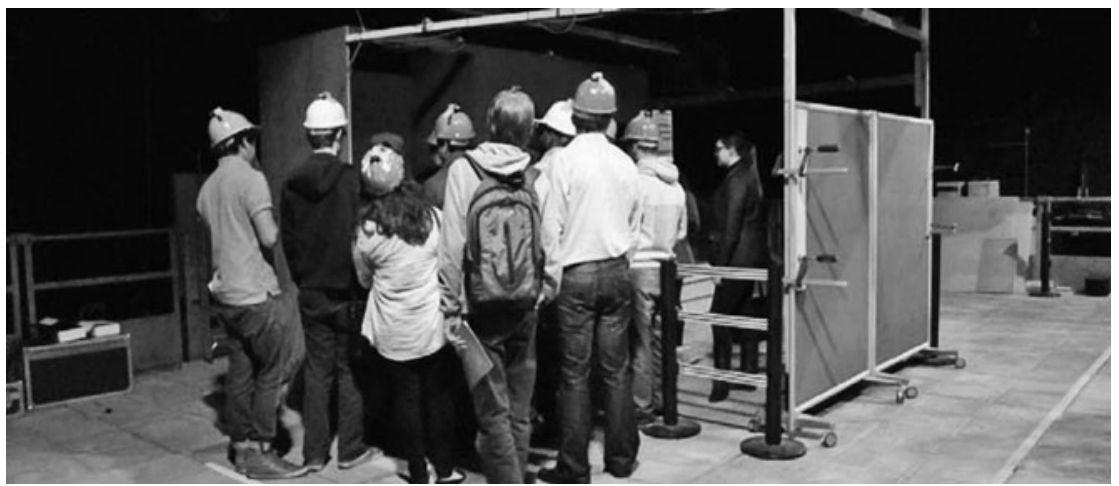

Appendix 9D Participants wearing motion trackers on hard hats
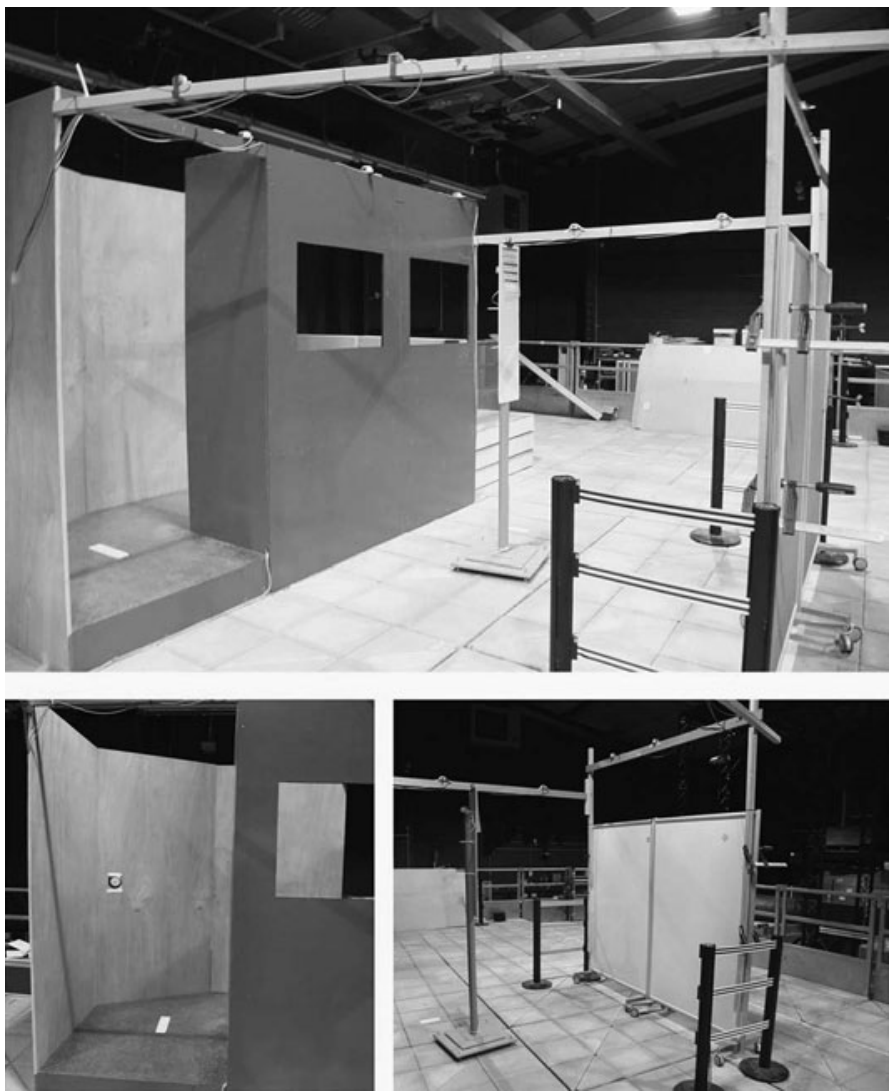

Appendix 9E Markers defining coordinate grids, within which motion trackers' positions are recorded are set-up around the experimental environments 


\section{Acknowledgements}

The author would like to thank Nick Calvert for help with the data analysis, and the UCL Accessibility Research Group for help with the experiment, as well as all participants who took part. Funding for this project was provided by the Engineering and Physical Sciences Research Council Grant no: EP/G037264/1 as part of UCL's Security Science Doctoral Training Centre.

\section{References}

Antonini, G., Venegas, S., Thiran, J., Bierlaire, M. (2004) A Discrete Choice Pedestrian Behavior Model for Pedestrian Detection in Visual Tracking Systems. RO 200409 09, proceeding; TRANSP-OR-REPORT-2004-001.

Baguley, T. (2012) Serious Stats: A Guide to Advanced Statistics for the Behavioral Sciences. Basingstoke: Palgrave Macmillan.

Beller, A., Garelik, S. and Cooper, S. (1980) Sex Crimes in the Subway. Criminology, 18(1), 35-52.

Brand, S., and Price, R. (2000) The Economic and Social Costs of Crime. London, Home Office Online Report 30/05.

British Transport Police (2011) Give Them an Inch and They'll Take All They Can. http://www.btp.police.uk. Available at: http://www.btp.police.uk/pickpockets/, accessed 7 August 2012.

Cepolina, E., and Tyler, N. (2005) Understanding Capacity Drop for Designing Pedestrian Environments. Available at: http://discovery.ucl.ac.uk/1412/1/Cepolina Tyler_paper_Walk21.pdf, accessed August 30, 2013.

Davies, A. C., and Velastin, S. A. (2005) A Progress Review of Intelligent CCTV Surveillance Systems. In: IEEE Intelligent Data Acquisition and Advanced Computing Systems: Technology and Applications; 05-07 Sept 2005, Sofia, Bulgaria. Eck, J. E., and Liu, L. (2008) Contrasting Simulated and Empirical Experiments in Crime Prevention. Journal of Experimental Criminology, 4(3), 195-213.

Ekblom, P. (1995) Less Crime, by Design. Annals of the American Academy of Political and Social Science, 539(1), 114-129.

Evans, G. (2009) Accessibility, Urban Design and the Whole Journey Environment. Built Environment, 35(3), 366-385.

Felson, M., and Cohen, E. L. (1979) Social Change and Crime Rate Trends: A Routine Activity Approach. American Sociological Review, 44(4), 588-608.

Fernández, Rodrigo, Pablo Zegers, Gustavo Weber, and Nick Tyler (2010) Influence of Platform Height, Door Width, and Fare Collection on Bus Dwell Time. Transportation Research Record: Journal of the Transportation Research Board, 2143(1), 59-66.

Fruin, J. (1992) Designing for Pedestrians. Public Transportation. United States. Available online [http://ntl.bts.gov/DOCS/11877/Chapter_8.html].

Fujiyama, T., Childs, C. R., Boampomg, D. and Tyler, N. (2005) Investigation of Lighting Levels for Pedestrians: Some questions about Lighting Levels of Current Lighting Standards. Walk 21-VI, Everyday Walking Culture, The 6th International Conference on Walking in the 21st Century, 22-23 September 2005, Zurich, Switzerland.

Helbing, D., Molnar, P., Farkas, I. J., and Bolay, K. (2001) Self-Organizing Pedestrian Movement. Environment and Planning B: Planning and Design, 28(3), 361-383. 
Helbing, D. et al. (2005) Self-Organized Pedestrian Crowd Dynamics: Experiments, Simulations, and Design Solutions. Transportation Science, 39(1), 1-24.

Helbing, D., and Balietti, S. (2011) How to Do Agent-Based Simulations in the Future: From Modeling Social mechanisms to Emergent Phenomena and Interactive Systems Design. SFI Working Paper: 11-06-024; Available online http://www. santafe.edu/media/workingpapers/11-06-024.pdf.

Holmes, N. P., and Spence, C. (2004) The Body Schema and the Multisensory Representation(s) of Peripersonal Space. Cognitive processing, 5(2), 94-105.

Hoogendoorn, S. P., and Daamen, W. (2005) Pedestrian Behavior at Bottlenecks. Transportation Science, 39(2), pp.147-159.

Jacoby, J., Johar, G. and Morrin, M. (1998) Consumer Behavior: A Quadrennium. Annual Review of Psychology, 49(1), 319-344.

Kabundi, M., and Normandeau, A. (1987) Crime in the Montreal Subway. International Criminal Police Review, 42(406), 24-27.

Kenney, D. J. (1986) Crime on the Subways: Measuring the Effectiveness of the Guardian Angels. Justice Quarterly, 3(4), 481-496.

King, B. G. (1948) Measurements of Man for Making Machinery. American Journal of Physical Anthropology, 6(3), 341-351.

Landon, E. (1971) Order Bias, the Ideal Rating, and the Semantic Differential. Journal of Marketing Research, 8(3), 375-378.

Liggett, R., Loukaitou-Sideris, A. and Isekl, H. (2001) Bus Stop-Environmental Connection: Do Characteristics of the Built Environment Correlate with Bus Stop Crime ? Transportation Research Record, Paper No. 0(613), 20-27.

Loukaitou-Sideris, A. (1999) Hot Spots of Bus Stop Crime: The Importance of Environmental Attributes. Journal of the American Planning Association, 65(4), 395-411.

Loukaitou-Sideris, A. (2012) Safe on the Move: The Importance of the Built Environment In: Ceccato, V. (ed) The Urban Fabric of Crime and Fear. New York, Dordrecht, London: Springer, 85-110.

Loukaitou-Sideris, A. and Liggett, R. (2000) On Bus-Stop Crime. ACCESS, 16, 18-21.

Loukaitou-Sideris, A. et al. (2001) Measuring the Effects of Built Environment on Bus Stop Crime. Environment and Planning B: Planning and Design, 28(2), 255-280.

Macal, C. M., and North, M. J. (2010) Tutorial on Agent-Based Modelling and Simulation. Journal of Simulation, 4(3), 151-162. Available at: http://www.palgravejournals.com/doifinder/10.1057/jos.2010.3, accessed 12 July 2012.

Macintyre, S., and Ross, H. (1996) Danger on the Dance Floor: A Study of the Interior Design, Crowding and Aggression in Nightclubs. In: Ross, H. (ed.) Policing for Prevention: Reducing Crime, Public Intoxication, and Injury. In Crime Prevention Studies, Vol. 7. Monsey, NY: Criminal Justice Press: Criminal Justice Press.

Metropolitan Police (2011) Pickpockets Targeted in Transport Crackdown. Available at: http://content.met.police.uk/News/Pickpockets-targeted-in-transport-crackdo wn/1400004504624/1257246745756, accessed 5 August 2012.

Moussaïd, M. et al. (2009) Experimental Study of the Behavioural Mechanisms Underlying Self-Organization in Human Crowds. Proceedings. Biological Sciences/ The Royal Society, 276(1668), 2755-2762.

Scholl, H. J. J. (2001) Agent-Based and System Dynamics Modeling: A Call for Cross Study and Joint Research, Proceeding HICSS '01: Proceedings of the 34th Annual Hawaii International Conference on System Sciences (HICSS-34)-Volume 3 Volume 3 Page 3003 IEEE Computer Society Washington, DC, USA.

Schultz, P. W., and Tabanico, J. J. (2009) Criminal Beware: A Social Norms Perspective on Posting Public Warning Signs. Criminology, 47(4), 1201-1222. 
Seyfried, A. et al. (2009) New Insight into Pedestrian Flow through Bottlenecks. Transportation Science, 43(3), 395-406.

Shellow, R., Romualdi, J. P. and Bartel, E. W. (1974) Crime in Rapid Transit Systems: An Analysis and a Recommended Security and Surveillance System. Transportation Research Record, 487, 1-12.

Smith, M. J., and Clarke, R. V. (2000) Crime and Public Transport. Crime and Justice, 27, 169-233.

Stewart, D. W., and Martin, I. M. (1994) Intended and Unintended Consequences of Warning Messages: A Review and Synthesis of Empirical Research. Journal of Public Policy and Marketing, 13(1), 1-19.

Teknomo, K. (2002) Microscopic Pedestrian Flow Characteristics: Development of an Image Processing Data Collection and Simulation Model. Doctoral Dissertation. Tohoku University.

Transport for London (2006) Accessible Bus Stop Design Guidance (January), 1-64. Available at: http://www.tfl.gov.uk/cdn/static/cms/documents/accessibile-busstop-design-guidance.pdf [Accessed 31st March 2015).

Transport for London (2011) Travel in London: Report 4. Available at: http://www. tfl.gov.uk/assets/downloads/corporate/travel-in-london-report-4.pdf, accessed 14 August 2012.

Transport for London (2012) 2011/2012 Crime Statistics Bulletin. Available at: http:// www.tfl.gov.uk/assets/downloads/corporate/crime-statistic-bulletin-201112.pdf, accessed 24 July 2012.

Wijermans, N. et al. (2007) Modelling Crowd Dynamics: Influence Factors Related to the Probability of a Riot. In: Proceedings of the Fourth European Social Simulation Association Conference (ESSA), Toulouse University of Social Sciences 531-541.

Wogalter, M. S., and Laughery, K. R. (1985) Behavioural Effectiveness of Warnings. In: Proceedings of the Human Factors Society 29th Annual Meeting, Santa Monica, CA Human Factors and Ergonomics Society, 679-683.

Yavuz, N., Welch, E.W. and Sriraj, P. S. (2007) Individual and Neighborhood Determinants of Perceptions of Bus and Train Safety in Chicago, Illinois: Application of Hierarchical Linear Modeling. Transportation Research Record, 2034(1), 19-26.

Except where otherwise noted, this chapter is licensed under a Creative Commons Attribution 4.0 Unported License. To view a copy of this license, visit http://creativecommons.org/licenses/by/4.0/ 